\title{
Massive and dense cores: the maternities of massive stars
}

\author{
Guido Garay \\ Departamento de Astronomía, Universidad de Chile, Santiago, Chile \\ email: guido@das.uchile.cl
}

\begin{abstract}
Massive stars are known to be formed in clusters. To understand their birth process it is essential to know the physical conditions of the parental cloud which is thought to play a critical role in determining the formation mechanism. In this contribution I summarize recent results from observations of dust, molecular and ionized gas emission we have made toward several massive star forming regions in the southern hemisphere. These observations are providing key evidence concerning the initial conditions for the formation of cluster of massive stars, allowing to characterize the physical properties of massive and dense cores and permitting to identify them in different stages of their early evolution.
\end{abstract}

Keywords. Stars: formation, ISM: clouds, radio continuum: ISM

\section{Introduction}

The formation processes and initial evolutionary phases of massive star birth are still not well understood (e.g., Garay \& Lizano 1999). Current questions in the field include: How are massive stars formed? Where are massive stars formed? Are massive protostars surrounded by disks? Do massive stars undergo a collimated bipolar outflow phase? As such, much observational effort has been performed during the last decade to investigate the early stages of high-mass star formation.

Surveys of emission in molecular lines excited at high temperatures and densities (Mauersberger et al. 1986; Cesaroni et al. 1992, 1994) have shown that the presence of hot and dense molecular cores (HMC's) is a common phenomenon towards massive star forming regions. The emerging consensus is that HMC's correspond to the cradles of massive stars, signalizing thus their birth place. HMC's are characterized by having diameters $<0.1 \mathrm{pc}$, temperatures $>100 \mathrm{~K}$, densities $>10^{7} \mathrm{~cm}^{-3}$ and luminosities in the range $10^{4}-10^{6} L_{\odot}$ (Kurtz et al. 2000; Cesaroni, this meeting). Whether the bulk of the luminosity is due to the accretion process or due to nuclear burning by the massive protostar is not well determined. These surveys raised the new questions of where are hot molecular cores found and how are they formed.

Understanding the formation and early evolution of massive stars requires knowledge of the physical conditions of their birth environment. In particular, observations of the HMC environment at spatial scales of parsecs may hold the key to distinguish between different modes of HMC formation, such as fragmentation, competitive accretion or coalescence. In this contribution, I will summarize the extensive observational effort carried out during the last few years by the Star Formation Group at the Universidad de Chile that have shed light on the processes taking place in the formation of high-mass stars and on the initial conditions for the formation of HMC's. 


\section{The maternities of massive stars}

In this section we discuss the observed and derived properties of the maternities of massive stars, namely of the molecular structures giving birth to clusters of massive stars. Several systematic studies have been carried out during the last decade to determine the characteristics of massive star forming regions (MSFR's) using as probe molecular line emission from high density tracers (Plume et al. 1992, 1997; Juvela 1996; Shirley et al. 2003). Since the properties of molecular cores derived from molecular line observations are likely to depend on the tracer used, we (Faúndez et al. 2004) made a survey of 1.2 millimeter dust continuum emission toward 146 IRAS sources thought to harbor highmass star forming regions. The objects were selected from the Bronfman et al. (1996) list of IRAS point sources with colors typical of ultra compact H II regions (Wood \& Churchwell 1989) and $\mathrm{CS}(2 \rightarrow 1)$ emission. Regions of $\sim 15^{\prime} \times 10^{\prime}$, centered on the IRAS sources, were mapped with an angular resolution of $\sim 24^{\prime \prime}$, using the SIMBA bolometer array on the SEST telescope. 1.2 millimeter emission was detected toward all IRAS sources. Other surveys of dust continuum emission toward large samples of high-mass star forming regions have been recently reported by Beuther et al. (2002), Mueller et al. (2002), and Williams et al. (2004).

We find that the dust cores harboring recently formed massive stars have typically sizes of $0.4 \mathrm{pc}$, masses of $5 \times 10^{3} \mathrm{M}_{\odot}$, densities of $2 \times 10^{5} \mathrm{~cm}^{-3}$, dust temperatures of $32 \mathrm{~K}$ and luminosities of $2 \times 10^{5} \mathrm{~L}_{\odot}$. A comparison with values derived from molecular line observations (e.g., Cesaroni et al. 1991, Juvela 1996, Plume et al. 1997) shows good agreement. For instance, from a survey of molecular emission, in several transitions of CS, toward MSFR's associated with water masers, Plume et al. (1997) found that high-mass stars are formed in molecular cores with typical radii of $0.5 \mathrm{pc}$, densities of $8 \times 10^{5} \mathrm{~cm}^{-3}$, and virial masses of $4 \times 10^{3} \mathrm{M}_{\odot}$. Clearly the mm dust continuum and high density molecular line emissions are tracing the same structures. We conclude that massive stars are formed in regions of molecular gas and dust with distinctive physical characteristics, which we will refer to as massive and dense cores (MDC's). The molecular line observations further show that MDC's have remarkable wide line widths, typically 6 $\mathrm{km} \mathrm{s}^{-1}$. Most ( 90\%) MDC's are found located within giant molecular clouds, but a few appear as isolated structures. These are ideal places to investigate the processes taking place in the formation of massive stars.

The mechanism of formation of massive and dense cores is still poorly known. The morphologies of the MDC's may shed some light on this issue. Of the cores observed by Faúndez et al. (2004), 24\% are associated with filamentary structures, whereas $23 \%$ show clumpy morphologies. The common appearance of elongated structures suggests that gravitational instabilities in filaments are likely to be an important mechanism of core formation (Larson 1985; Tilley \& Pudritz 2003). The clumpy structure could be explained if the cores are formed by turbulent shocks (Padoan et al. 2001). Therefore, both gravitational fragmentation and turbulent shocks seem to play a fundamental role for the origin of the massive and dense cores.

Several studies have shown that significant correlations exist between the line width, size and density of molecular clouds (cf. Larson 1981, Myers 1983). Do MDC's follow the same relationships? Fig. 1 plots the line width versus size (left panel) and the density versus size (right panel) relationships for massive and dense cores. The data for MDC's have been taken from Plume et al. (1997), Shirley et al. (2003), and Garay et al. (2005). For comparison also plotted are the values observed for dense cores in low-mass clouds (Myers 1983). Fig. 1 clearly shows that although correlations exists between the parameters of MDC's, the relationships are not the same for the different type of cores. At a 

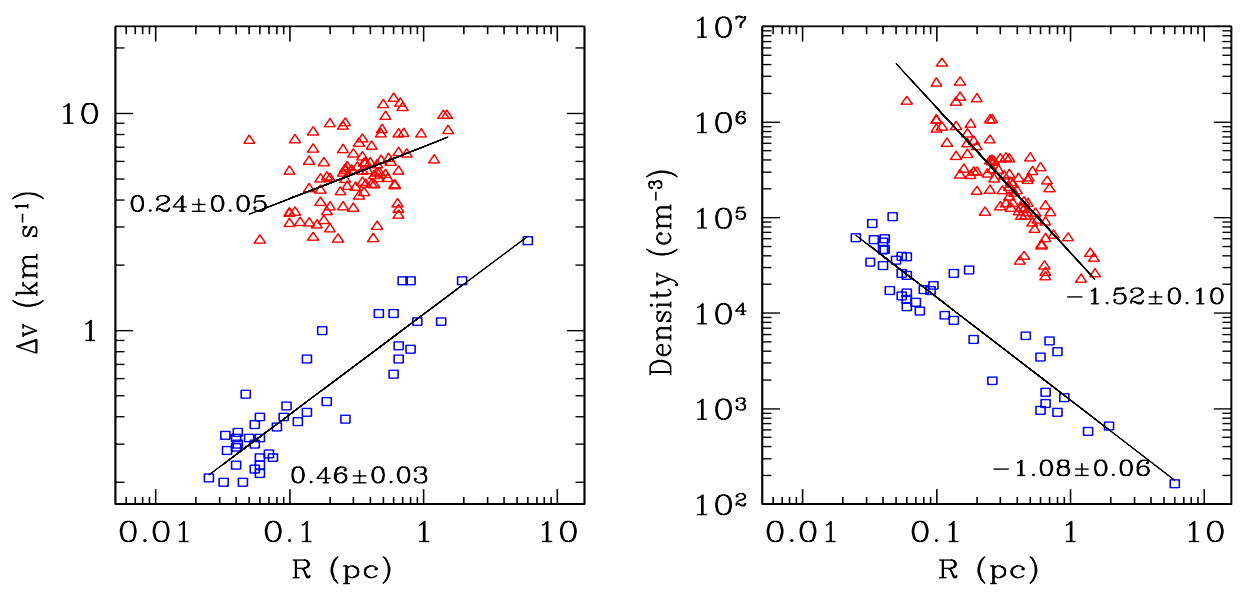

Figure 1. Correlations between physical parameters of molecular cores. Left: Line width versus radius. Right: Density versus radius. Squares: Dense cores in dark clouds. Data from Myers (1983). Triangles: Massive and dense cores. Lines are least squares linear fits to the data points.

given size, massive and dense cores have much broader line widths than cores in lowmass clouds. The physical basis for the line width versus size relationship is still poorly understood. Fuller \& Myers (1992) concluded that the observed relation for low-mass cores within dark clouds reflects the initial conditions for the formation of low-mass stars rather than being a consequence of star-core interaction. The different line width-size relationships for the different type of cores may thus reflect differences in their initial physical conditions. Assuming that the cores are in virial equilibrium, the shift in line width at a fixed cloud size could be explained as due to differences in their molecular densities. The line widths of MDC's are roughly 7 times larger than those of low-mass cores, which would imply, if virialized, that their molecular densities are typically greater by a factor of 50 (as illustrated in the right panel of Fig. 1). It is worth mentioning at this point that the densities derived from molecular line analysis for a few MDC's are in good agreement with those implied by the virial hypothesis.

We note that the above explanation simply recasts the problem of the shift in the $\Delta v$ versus $R$ relationship to the problem of what produces the high densities in MDC's. Larson (1981) suggested that the systematic differences in line width among the different types of cores may represent different amounts of evolution away from a "primordial" state of turbulence represented most closely by the low-mass cores. The more massive cores may have experienced more gravitational contraction than the low-mass cores, which would increase their virial velocities. We caution that it is not possible to infer from Fig. 1 that there is a "bimodal" distribution in core properties. It is possible that when all type of dense cores are considered they will fill the $\Delta v$ vs $R$ and $n$ vs $R$ spaces.

To assess the dynamical state of MDC's, Mardones (2003) analyzed the $\operatorname{CS}(2 \rightarrow 1)$ line profiles from a sample of 639 southern objects $\left(\delta<-20^{\circ}\right)$ observed by Bronfman et al. (1996). He found that the bulk of the line profiles are fairly symmetric, suggesting that most MDC's appear to be stable against gravitational collapse. This is supported by the fact that the virial parameter $\alpha=0.9 \Delta v^{2} R / G M$, where $\Delta v$ is the FWHM line width and $M$ the mass determined from the dust observations is $\sim 1$ for most MDC's, implying that they are in virial equilibrium. The observed line widths, of $\sim 6 \mathrm{~km} \mathrm{~s}^{-1}$, are much greater than the thermal widths $\left(\sim 0.2 \mathrm{~km} \mathrm{~s}^{-1}\right.$ at kinetic temperatures of $\left.32 \mathrm{~K}\right)$ 
implying that a considerable amount of non-thermal support is required. The question of the mechanism of support against collapse is related to the question of What is the origin of the broad line widths in MDC's? Most likely, the broad line widths are due to the presence of turbulent gas motions, which are believed to consist mainly of magnetohydrodynamic waves. The mean pressures associated to the turbulent motions are very high, typically $\sim 3 \times 10^{8} \mathrm{~K} \mathrm{~cm}^{-3}$. The large amount of support provided by the turbulent pressure allows the existence of cores in hydrostatic equilibrium with much larger densities than in low-mass cores. Mardones (2003) also found wing emission towards $15 \%$ of the MDC's, showing that bipolar outflow is a common phenomenon in the formation process of massive stars (Beuther et al. 2002; Shepherd, this meeting). Outflow velocities in the $\mathrm{CS}(2 \rightarrow 1)$ lines are typically $15 \mathrm{~km} \mathrm{~s}^{-1}$. Finally, he found self-absorbed profiles in about $8 \%$ of the sources; half of them being indicative of overall expansion motions and the other half indicative of inward motions.

\section{Massive and dense cold cores}

Because of the selection criteria used, most of the MDC's in the Faúndez et al. (2004) sample, as well as MDC's in other samples, are associated with luminous objects, implying that massive stars have already formed within them. The fast stellar winds and strong ionizing radiation from the young massive star (or stars) are expected to have a profound impact on the MDC from which they form, drastically changing its internal structure and chemistry and ultimately destroying it.

The question arises then as to whether or not the physical conditions of the MDC's with embedded young massive stars are representative of the large scale (few tenths of parsecs) initial conditions for the formation of massive stars. To address this question requires the search of massive and dense cores in the earliest stage of evolution, previous to the formation of a central massive object. These cores should be characterized by having similar densities and sizes as massive dense cores with embedded high-mass stars, but lower luminosities and cooler temperatures $\left(T_{d}<15 \mathrm{~K}\right)$. The bulk of their low luminosity is expected to be emitted in the (sub)millimeter range. Massive cores in early stages of evolution are likely to be below the detection limits of IRAS and MSX and to find them we need to resort to $\mathrm{mm} / \mathrm{sub}-\mathrm{mm}$ observations.

Since the sites of massive star formation are known to be gregarious, a further goal of our 1.2-mm dust continuum survey (Faúndez et al. 2004) was to find massive starless cores in the vicinity of cores associated with IRAS sources. We searched for millimeter sources without counterparts at mid infrared (MSX) and far infrared (IRAS) wavelengths, detecting a handful of candidates (Garay et al. 2004). The lack of detections in the IRAS bands makes it impossible the determination of the dust temperature, but indicates an upper limit of $\sim 17 \mathrm{~K}$. These cold dust cores have radii in the range $0.2-0.3 \mathrm{pc}$, masses in the range $4 \times 10^{2}-2 \times 10^{3} M_{\odot}$ and densities of $\sim 2 \times 10^{5} \mathrm{~cm}^{-3}$. The confirmation that these objects are truly massive starless cores requires molecular line observations. We have made CS line observations toward most of these candidates, and found that their physical characteristics (masses, sizes and densities) are similar to those of the cores with embedded massive stars, indicating that they can be genuinely associated with the initial conditions of massive star formation. In particular, their linewidths are broad $(\sim 4 \mathrm{~km}$ $\mathrm{s}^{-1}$ ) which is only characteristic of massive dense cores. The virial masses derived from the linewidth of CS observations are in agreement with the masses estimated from the dust emission. We suggest that these massive and dense cold cores will eventually collapse to form high-mass stars, and that their physical conditions are representative of the initial conditions for the formation of massive stars. 


\section{Multiwavelength studies of a selected sample of MDC's}

We are currently studying in detail a sample of 18 luminous IRAS point sources in the southern hemisphere thought to be representative of young massive dense cores. The sources were selected from the list of Bronfman et al. (1996), by requiring that they have IRAS luminosities greater than $2 \times 10^{4} \mathrm{~L}_{\odot}$ (implying that they contain at least one embedded massive star) and by having line profiles indicative of either inward or outward motions and in some cases broad wings. This database is expected to contain MSFR's in different evolutionary stages, ranging from pre-UCHII cores to cores with UCHII regions. Our study incorporates radio continuum data at four frequencies (1.4, 2.5, 4.8 and $8.6 \mathrm{GHz}$ ) obtained with the Australia Telescope Compact Array (ATCA), 1.2-mm continuum emission data obtained using the SIMBA bolometer array at SEST, a series of SEST multi-molecule and multi-transitions between 85 and $250 \mathrm{GHz}\left(\mathrm{CS}, \mathrm{C}^{34} \mathrm{~S}\right.$, $\mathrm{HCO}^{+}, \mathrm{H}^{13} \mathrm{CO}^{+}, \mathrm{C}^{18} \mathrm{O}$, SiO, SO and $\left.\mathrm{H}_{2} \mathrm{~S}\right)$, and mid-infrared data (N11.9 $\mu \mathrm{m}$ filter) obtained using TIMMI2 on the 3.6-m telescope in La Silla. Dust emission (at 1.2-mm and $11.9 \mu \mathrm{m}$ ) and molecular emission in all lines were detected towards all MDC's.

Where are massive stars formed within the massive dense cores? This is an important issue because their location is likely to be a useful indicator of the formation mechanism. Since MDC's have large column densities, typically $5 \times 10^{23} \mathrm{~cm}^{-2}$, the probe must penetrate deeply into the core. Our radio continuum observations with high angular resolution are an ideal probe. They show the presence of UC H II regions in most (15/18) of the MDC's, implying that they have ongoing massive star formation as already indicated by their high luminosities. A comparison of the radio images with 1.2-mm continuum images show that the UC H II regions are usually located at the peak of the dust continuum emission. Young massive stars appear, thus, to form at the center of MDC's.

Among our sample we found two luminous MDC's that are undetected at radio wavelengths and a third for which the radio emission is much smaller than that expected from its bolometric luminosity (Garay et al. 2002, 2003). These three MDC's are likely to be in early evolutionary stages, prior to the appearance of ultra compact regions of ionized gas. They all have line emission profiles indicative of large scale inflow motions. The spectra in optically thick lines $\left(\mathrm{CS}\right.$ and $\left.\mathrm{HCO}^{+}\right)$show double-peaked line profiles, with a bright blue-shifted peak, whereas the profiles of optically thin lines $\left(\mathrm{C}^{34} \mathrm{~S}\right.$ and $\left.\mathrm{H}^{13} \mathrm{CO}^{+}\right)$ show an approximately symmetric single component with a peak center velocity located in between the two peaks. These spectroscopic signatures suggest that the bulk of the molecular gas in these cores is undergoing large-scale inward motions. The derived inward speeds are typically $0.5 \mathrm{~km} \mathrm{~s}^{-1}$, smaller than the free-fall velocity expected at the outer radius of the cores, suggesting that the collapse is not dynamical. The mass infall rate associated with the large scale inflow of matter is of the order of $10^{-2} M_{\odot} \mathrm{yr}^{-1}$. These findings suggest that massive stars are formed under very high mass accretion rates and that the expansion of the (proto) HII region is quenched by mass loading (e.g., Osorio, Lizano \& D'Alessio 1999).

In what remains I concentrate the discussion on one of the most interesting sources: IRAS 16547-4247, which has a bolometric luminosity of $6.2 \times 10^{4} L_{\odot}$, equivalent to that of a single young $\mathrm{O} 8$ star. The $1.2-\mathrm{mm}$ dust continuum observations indicate that this MDC has a diameter of $0.4 \mathrm{pc}$ and a mass of $1.3 \times 10^{3} M_{\odot}$. The radio continuum observations show a well collimated triple radio source, centered on the $1.2-\mathrm{mm}$ continuum emission peak. The central radio source corresponds to a thermal jet, whereas the outer nonthermal components correspond to regions of shocked gas at the working surfaces of the jet (Garay et al. 2003). Also seen towards this MDC is a chain of $\mathrm{H}_{2} 2.12 \mu \mathrm{m}$ emission knots that trace a large scale collimated flow, extending over $1.5 \mathrm{pc}$, most likely driven 
by the thermal jet (Brooks et al. 2003). These observations indicate that IRAS 165474247 harbors the most massive and luminous embedded young stellar object known to be associated with jet and collimated outflow phenomena. This finding supports the idea that massive stars in the process of formation pass through similar evolutionary phases than low-mass stars, including the formation of disks and the fast ejection of matter along the poles. The observations of this luminous object provide the best evidence to date in support for an accretion model of massive star formation.

\section{Acknowledgements}

I gratefully acknowledge support from the Centro de Astrofísica FONDAP No. 15010003.

\section{References}

Beuther, H., Schilke, P., Menten, K., Motte, F., Sridharan, T. K. \& Wyrowski, F. 2002, ApJ, 566,945

Bronfman, L., Nyman, L-A. \& May, J. A. 1996, A\&AS, 115, 81

Brooks, K. J., Garay, G., Mardones, D. \& Bronfman, L. 2003, ApJ, 594, L131

Cesaroni, R., Walmsley, C.M., Kömpe, C. \& Churchwell, E. 1991, A\&A, 252, 278

Cesaroni, R., Walmsley, C.M. \& Churchwell, E. 1992, A\&A, 256, 618

Cesaroni, R., Churchwell, E., Hofner, P., Walmsley, C.M. \& Kurtz, S. 1994, A\&A, 288, 903

Faúndez, S., Bronfman, L., Garay, G., Chini, R. May, J. \& Nyman, L.A. 2004, A\&A, 426, 97

Fuller, G.A. \& Myers, P.C. 1992, ApJ, 384, 523

Garay, G. \& Lizano S. 1999, PASP, 111, 1049

Garay, G., Brooks, K., Mardones, D., Norris, R. P. \& Burton, M.G. 2002, ApJ, 579, 678

Garay, G., Brooks, K., Mardones, D. \& Norris, R. P. 2003, ApJ, 587, 739

Garay, G., Faúndez, S., Mardones, D., Bronfman, L., Chini, R. \& Nyman, L.-A. 2004, ApJ, 610, 313

Garay et al. 2005, in preparation

Juvela, M. 1996, A\&AS, 118, 191

Kurtz, S., Cesaroni, R., Churchwell, E., Hofner, P. \& Walmsley, C. M. 2000, in Protostars and Planets IV, eds Mannings, V., Boss, A.P., Russell, S.S. (Tucson: University of Arizona Press), p. 299

Larson, R.B. 1981, MNRAS, 194, 809

Larson, R.B. 1985, MNRAS, 214, 379

Mardones, D. 2003, in Open Issues in Local Star Formation, eds Lepine, J., Gregorio-Hetem, J. (Dordrecht: Kluwer), p. 359

Mauersberger, R., Henkel, C., Wilson, T.L. \& Walmsley, C.M. 1986 A\&A, 162, 199

Mueller, K., Shirley, Y., Evans, N. \& Jacobson, H. 2002, ApJS, 143, 469

Myers, P. C. 1983, ApJ, 270, 105

Osorio, M., Lizano S. \& D'Alessio P. 1999 ApJ, 525, 808

Padoan, P., Juvela, M., Goodman, A. \& Nordlund, A. 2001, ApJ, 553, 227

Plume, R., Jaffe, D. T. \& Evans, N. J. 1992 ApJS 78, 505

Plume, R., Jaffe, D. T., Evans, N. J., Martín-Pintado, J. \& Gómez-González, J. 1997, ApJ 476, 730

Shirley, Y.L., Evans, N.J. II, Young, K.E., Knez, C. \& Jaffe, D.T. 2003, ApJS, 149, 375

Tilley, D. \& Pudritz, R. 2003, ApJ, 593, 426

Williams, S.J., Fuller, G.A. \& Sridharan, T.K. 2004, A\&A, 417, 115

Wood, D.O.S. \& Churchwell, E. 1989, ApJS, 69, 831 